\title{
Avulsion et implantation immédiate
}

\section{Immediate implantation after extraction}

\section{Résumé}

\section{MOTS-CLES :}

- Extraction, implantation immédiate.

\section{KEYWORDS:}

- Extraction, immediate implantation.
L'intérêt pour l'avulsion implantation immédiate ne cesse de croître. Pour autant, les situations d'échec sont là pour nous rappeler qu'il ne faut pas trop vite banaliser cette technique.

L'indication de l'avulsion implantation immédiate doit être mûrement réfléchie et le succès reste très largement opérateur dépendant.
Abstroct

The interest for immediate implantation after extraction continues to increase. However, cases that have failed serve to remind us that this technique should not be taken for granted. Recourse to such a technique must be properly thought through and success depends mostly on the operator.
- Philippe LECLERCQ, Docteur en chirurgie dentaire,

Expert près la cour d'appel de Paris, 45, rue de Courcelles, 75017 Paris

Olivier GRANJON, Docteur en chirurgie dentaire.

Déborah PHILLIPS, Docteur en chirurgie dentaire.

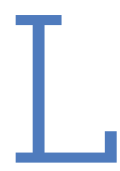

e développement de la chirurgie mini-invasive dans les différentes spécialités médicales et l'exigence croissante des patients ont conduit les chirurgiens dentistes à proposer des traitements avec avulsion dentaire et implantation dans le même temps opératoire.

Cette technique que nous nommerons dans cet article AII (avulsion implantation immédiate), par opposition à la technique conventionnelle où l'implantation est différée (AID), a pour intérêt de réduire le nombre d'interventions chirurgicales et de diminuer le temps du traitement.

L'AII a été le sujet de nombreuses études animales et cliniques montrant que l'ostéo-intégration de l'implant peut être obtenue avec un taux de succès équivalent à celui d'une technique conventionnelle. Cependant, la corrélation entre le succès implantaire et le succès prothétique n'est pas toujours vérifiée [1-3].

Il nous semble donc important de rappeler dans cet article que l'AII reste une technique à risque dont il faut respecter scrupuleusement les indications sous peine d'être confronté à des situations difficiles à gérer prothétiquement. Nous présenterons quelques cas cliniques illustrant les complications qui sont les plus fréquemment observées avec les pièges à éviter et ferons le point sur la mise en œuvre d'un protocole opératoire au travers de deux cas cliniques.

\section{LES SITUATIONS D'ÉCHEC ASSOCIÉES À L'AII \\ LES PLUS FRÉQUEMMENT RENCONTRÉES}

\section{All et récession gingivale (fig. 1,2 et 3 )}

Suite à l'avulsion dentaire, le stimulus physiologique de charge exercé par la dent naturelle disparaît, il s'ensuit une résorption de l'os alvéolaire en épaisseur et en hauteur.

Dans la région du maxillaire antérieur, cette résorption peut intéresser $40 \%$ à $60 \%$ du volume de l'os alvéolaire au cours des deux premières années. Elle peut alors compromettre le bon positionnement de l'implant et 

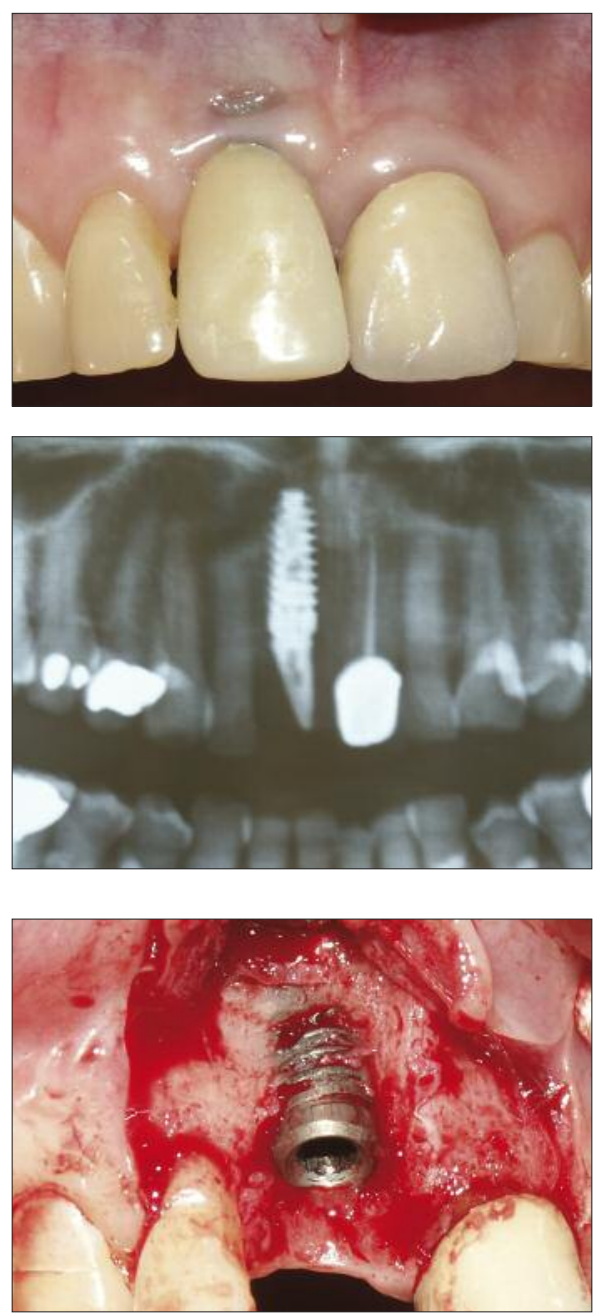

4 Fig. 3 :

Le traitement préconisé a été la dépose de l'implant, la réalisation d'une greffe osseuse et la mise en place d'un nouvel im-

plant à 4 mois.

Cette patiente a consulté suite à l'apparition récente d'une récession gingivale au niveau du col implantaire et souhaite corriger l'aspect inesthétique de l'élément prothétique. L’implant a été placé par un confrère selon un protocole d'AII.

La radiographie panoramique a été prise 8 mois après la mise en charge de l'implant avec une prothèse transitoire. gencive kératinisée sont des facteurs à prendre en compte. Dans la zone esthétique antérieure, une analyse attentive pré-opératoire mais également peropératoire est indispensable pour s'assurer de la faisabilité d'une telle technique. L'AII doit être contreindiquée dans les zones antérieures à parodonte fin. un critère d'exclusion pour la plupart des équipes

\section{All et complication infectieuse}

(fig. 4 et 5)

La présence d'une infection aiguë ou chronique est ayant publié dans la littérature scientifique.

Cependant, une expérimentation animale récente par Novaes et al. en 2004 conclut que les infections chroniques ne seraient pas une contre-indication absolue à l'AII si le curetage alvéolaire est réalisable et si les recommandations pré- et post-opératoires sont respectées [6,7].

Forts de cette expérience animale, certains praticiens aimeraient élargir le champ d'application de l'AII sur des dents présentant des foyers infectieux ou chez des patients atteints de parodontite terminale non contrôlée. Il nous semble que les connaissances biologiques fondamentales doivent nous inciter à la plus grande prudence vis-à-vis de ces travaux [8].

La microbiologie a montré que la flore pathogène présente dans la cavité buccale est très diversifiée avec parfois des germes résistants. Le curetage mécanique soigneux, l'utilisation d'antiseptique et d'antibiotique par le chirurgien ne suppriment en rien le rôle fondamental des cellules de l'hôte qui interviennent dans lélimination des réactions inflammatoires et infectieuses au cours de la cicatrisation.

La mise en place de l'implant immédiatement après le débridement de la lésion interfère avec le bon déroulement de ces réactions cellulaires et augmente le risque d'une complication infectieuse ou inflammatoire. Or, avec un protocole d'AII, les parois alvéolaires oblige le chirurgien à avoir recours à des aménagements osseux pré- ou per-opératoires.

Ceci a longtemps été un argument de poids en faveur de l'AII, présentée comme le moyen de maintenir le volume osseux alvéolaire après avulsion. L'implant placé immédiatement dans l'alvéole jouerait le rôle de « tuteur osseux » et empêcherait la résorption post-avulsion.

Des études récentes remettent en cause cette hypothèse. Boticelli et al. en 2004 observent que quelle que soit la technique utilisée, une résorption post-avulsion s'organise au cours des trois premiers mois de cicatrisation sans différence significative entre l'AII et l'AID $[4,5]$. Cette résorption est difficilement prédictible et peut saccompagner d'une récession gingivale qui compromet le succès esthétique de la réhabilitation prothétique. Cette résorption post-avulsion est dépendante de nombreux facteurs généraux et locaux.

\section{Facteurs généraux}

Le tabagisme, la mauvaise hygiène buccodentaire, les pathologies systémiques (diabète, déficit immunitaire, traitement immunosuppresseur, patient irradié...) doivent être considérés comme des contre-indications à cette technique.

\section{Facteurs locaux}

Le nombre et la nature des dents concernées, l'intégrité et l'épaisseur des parois alvéolaires, la présence d'une
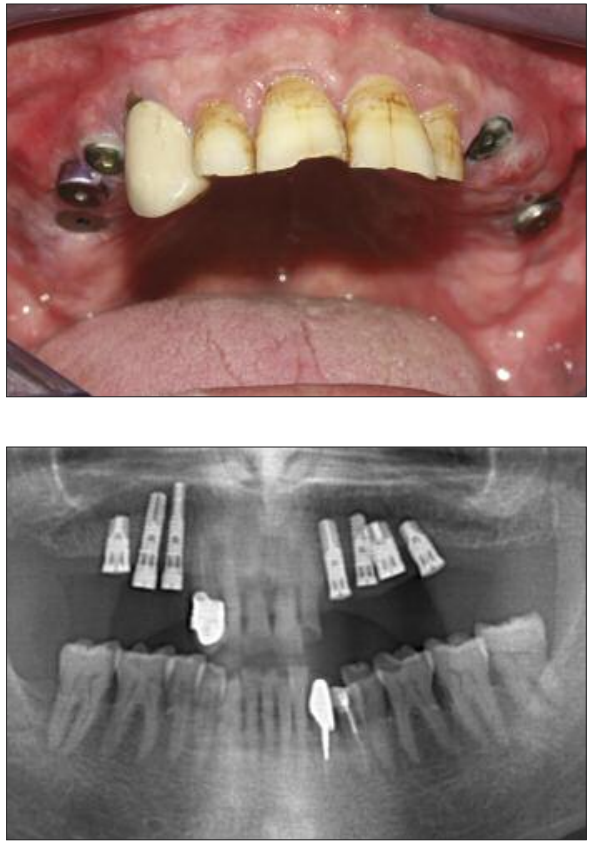

$\triangleleft$ Fig. 4 :

Ce patient a consulté trois mois après la pose des implants. Il se plaint de douleurs persistantes et la présence de suppurations autour des implants malgré la prise d'un traitement antibiotique (Augmentin'). L'anamnèse révèle que les molaires et prémolaires atteintes de lésions parodontales terminales ont été avulsées pour mettre en place des implants dans le même temps opératoire.

$\triangleleft$ Fig. 5 :

Radiographie panoramique prise un mois après l'intervention d'AII, les sept implants doivent être déposés. 
résiduelles sont fines et susceptibles de se résorber de façon importante. Le rapport bénéfice/risque de l'AII par rapport à une technique d'implantation différée nous semble alors franchement défavorable. Dans un site infecté, l'AII est contre-indiquée et ne peut être considérée comme faisant partie des connaissances avérées.

En présence d'une maladie parodontale, un prélèvement et une analyse au microscope de la flore buccale doivent être réalisés afin de déterminer si la maladie est contrôlée ou évolutive (présence de bactéries anaérobies, amibes...) [9]. Dans ce deuxième cas de figure, un traitement de décontamination, en suivant par exemple le protocole décrit par Marc Bonner, devra être mis en œuvre avant d'envisager l'AII.

\section{All et mauvais positionnement de l'implant (fig. 6 à 9)}

Après l'avulsion de la dent, la cavité alvéolaire a une forme plus ou moins ovoïde, un diamètre souvent supérieur à celui de l'implant et un axe différent du positionnement idéal de l'implant.

Au cours de l'AII, le forage sera un point clé, il a un double objectif.

\section{Obtenir la stabilité primaire de l'implant}

La présence d'au moins $4 \mathrm{~mm}$ d'os résiduel au-delà de l'apex est une condition sine qua non à l'obtention

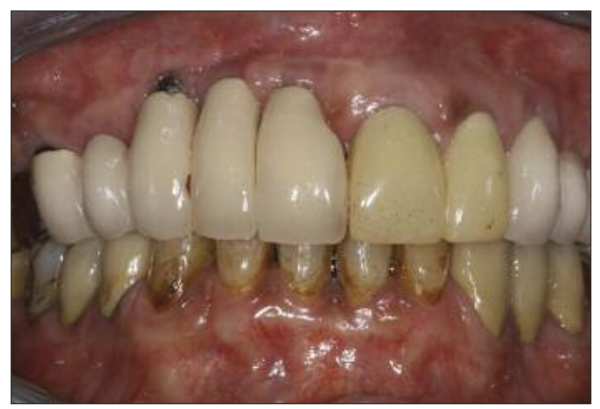

4 Fig. 6 :

La patiente consulte car elle n'est pas satisfaite du résultat esthétique de la réhabilitation implanto-portée compensant 11/12/13/14/15 réalisée deux ans auparavant. Les quatre implants supportant la prothèse ont été placés en même temps que lélimination de quatre organes dentaires.

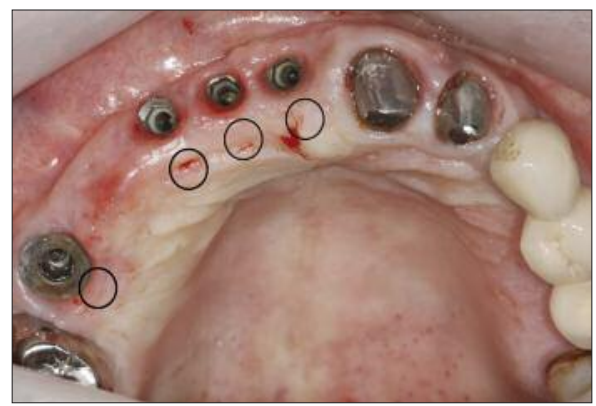

4 Fig. 7 :

Après dépose de la réhabilitation implanto-portée, il s'avère que les implants sont parfaitement ostéo-intégrés mais l'émergence et l'axe des implants sont excessivement vestibulés. Lémergence des implants devrait être située au niveau des cercles noirs pour pouvoir réaliser une réhabilitation esthétique. d'une stabilité primaire de l'implant. L'AII sera contre-indiquée pour les dents présentant un rapport étroit avec une structure noble tel que le nerf dentaire, les sinus maxillaires, les fosses nasales, les dents incluses.

Positionner l'implant selon les critères établis par Tarnow et Wallace [10]

Le bon positionnement de l'implant garantit le résultat esthétique de la réhabilitation prothétique sur le long terme.

Selon Tarnow et al., l'implant doit être positionné au moins à $2 \mathrm{~mm}$ du rebord vestibulaire et des dents adjacentes. Pour cela, l'implant doit être mis en place au contact de la paroi palatine ou linguale de l'alvéole.

Hwang et al. proposent la technique du guidage apicopalatin lors du forage du site implantaire pour éviter le glissement des forets en direction de la paroi vestibulaire. Le franchissement de la paroi palatine se fait avec une fraise boule de $2 \mathrm{~mm}$ de diamètre dans sa moitié apicale [11]. Ceci requiert une certaine expérience de la part du chirurgien. Les dents ectopiques, les dents pluri-radiculées sont des situations délicates à gérer et doivent être considérées comme des contre-indications relatives.

Lanalyse des situations déchec nous montre que le champ d'application de l'AII est finalement réduit.

\section{LE PROTOCOLE OPÉRATOIRE}

\section{Cas clinique $\mathrm{n}^{\circ} 1$ (fig. 10 à 22) et cas clinique $\mathrm{n}^{\circ} 2$ (fig. 23 à 30)}

Les situations cliniques pour lesquelles le protocole d'AII peut être discuté sont les dents présentant une carie radiculaire sous-gingivale, les dents présentant une rhysalyse externe ou interne, les dents fracturées, les échecs endodontiques définitifs, les dents lactéales et les apex dentaires résiduels. Le patient doit être conscient de la difficulté et des risques de ce protocole. Le praticien reste seul décisionnaire, si toutes les conditions nécessaires au succès thérapeutique ne sont pas réunies, il doit modifier le plan de traitement et avoir recours à une technique classique.

Voici une proposition de protocole qui se veut générale.

\section{Avulsion atraumatique}

Lavulsion est le premier temps opératoire. Elle conditionne le bon déroulement de l'intervention et doit être réalisée dans le respect de la préservation tissulaire.

\footnotetext{
Fig. 8 et 9 :

L'axe des implants est fortement projeté en vestibulaire. Afin de répondre aux exigences esthétiques de la patiente, la nouvelle réhabilitation prothétique a nécessité la dépose des implants, la réalisation d'une greffe osseuse, la mise en place de quatre nouveaux implants et une chirurgie mucco-gingivale. La dépose des implants a été réalisée grâce à la piézochirurgie et un tourne à gauche.
}
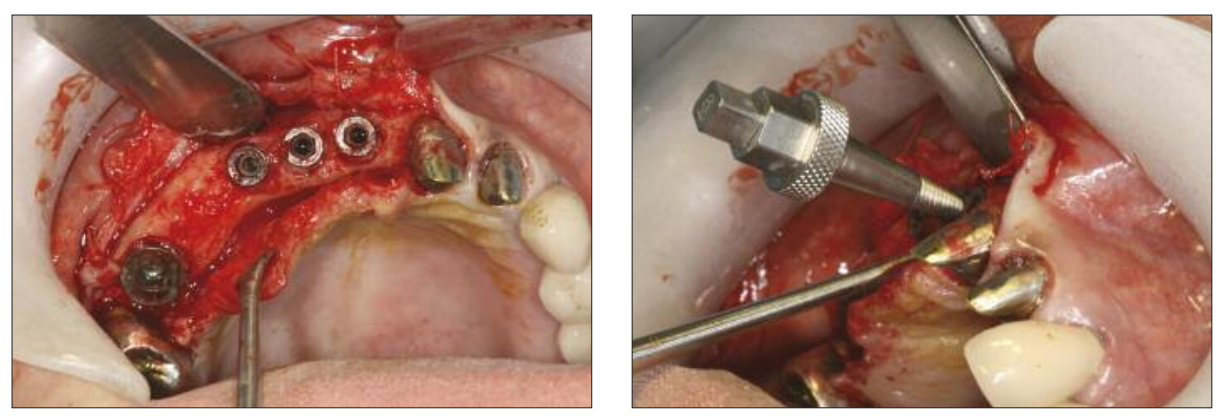
Il est recommandé de faire la syndesmotomie à l'aide d'un bistouri lame 15, de luxer la dent et de l'élever avec des instruments de type syndesmotomes de Bernard et périotomes. Dans la mesure du possible, il faudra éviter d'avoir recours à un davier qui entraîne des déformations et des lésions des parois alvéolaires. L'alvéole doit ensuite être curetée avec soins pour éliminer la présence éventuelle de tissus mous ou de granulation. Une fraise boule montée sur contre-angle peut être utilisée à cet effet.

\section{Temps chirurgical implantaire}

Il n'y a pas de consensus concernant la levée d'un lambeau. Certains praticiens préconisent de ne pas réaliser de lambeau afin de préserver la vascularisation périostée de la table osseuse vestibulaire [12].

Toutefois, un lambeau sans décharge et d'amplitude minimale peut s'avérer nécessaire. Il permettra d'avoir une bonne visualisation du site implantaire et de réduire ainsi le temps de l'intervention, d'estimer avec précision la dimension de la crête osseuse et la proximité des dents adjacentes et par-dessus tout de visualiser l'axe implantaire.

Il sensuit une séquence de forage et la mise en place de l'implant. Cette séquence de forage pourra être adaptée de façon à sous-calibrer l'alvéole implantaire pour augmenter la stabilité primaire. Elle devra être mise en œuvre selon le guidage apico-palatin décrit par Hwang et al. afin de positionner l'implant en position palatine dans l'alvéole.

Concernant la gestion du hiatus entre l'implant et la crête alvéolaire, il n'y a pas non plus de consensus sur l'utilité de combler l'espace implant-crête ou la nécessité de réaliser une régénération tissulaire guidée [13]. Il semblerait, en effet, que le caillot à lui seul permettrait le remplissage de cet espace vide par de los néoformé et qu'il n'est pas nécessaire de rechercher le recouvrement de l'implant par déplacement du lambeau.

Nous ne traiterons pas dans cet article de la temporisation, ni de la mise en charge immédiate éventuelle. Néanmoins, il nous semble utile de rappeler que, dans la littérature, les conditions du succès sont : la stabilité primaire et l'absence de micromouvement de l'implant pendant toute la phase de cicatrisation initiale $[14,15]$. Ainsi, il est nécessaire de choisir une temporisation adaptée sans sollicitation mécanique excessive.

\section{Conseils post-opératoires}

Le patient est désormais lui-même acteur du succès de l'intervention. Il faudra l'informer du caractère impérieux de n'exercer aucune contrainte ou charge sur l'implant. Il devra, pendant 6 à 8 semaines, adapter son régime alimentaire.

Une hygiène rigoureuse devra être observée et la prescription de complément au brossage comme des bains de bouche est fortement recommandée.

\section{Cas clinique $\mathrm{n}^{\circ} 1$}

$>$ Fig. 10 et 11 :

Situation initiale, présence d'une carie radiculaire sous-gingivale au niveau de la racine de 24 .
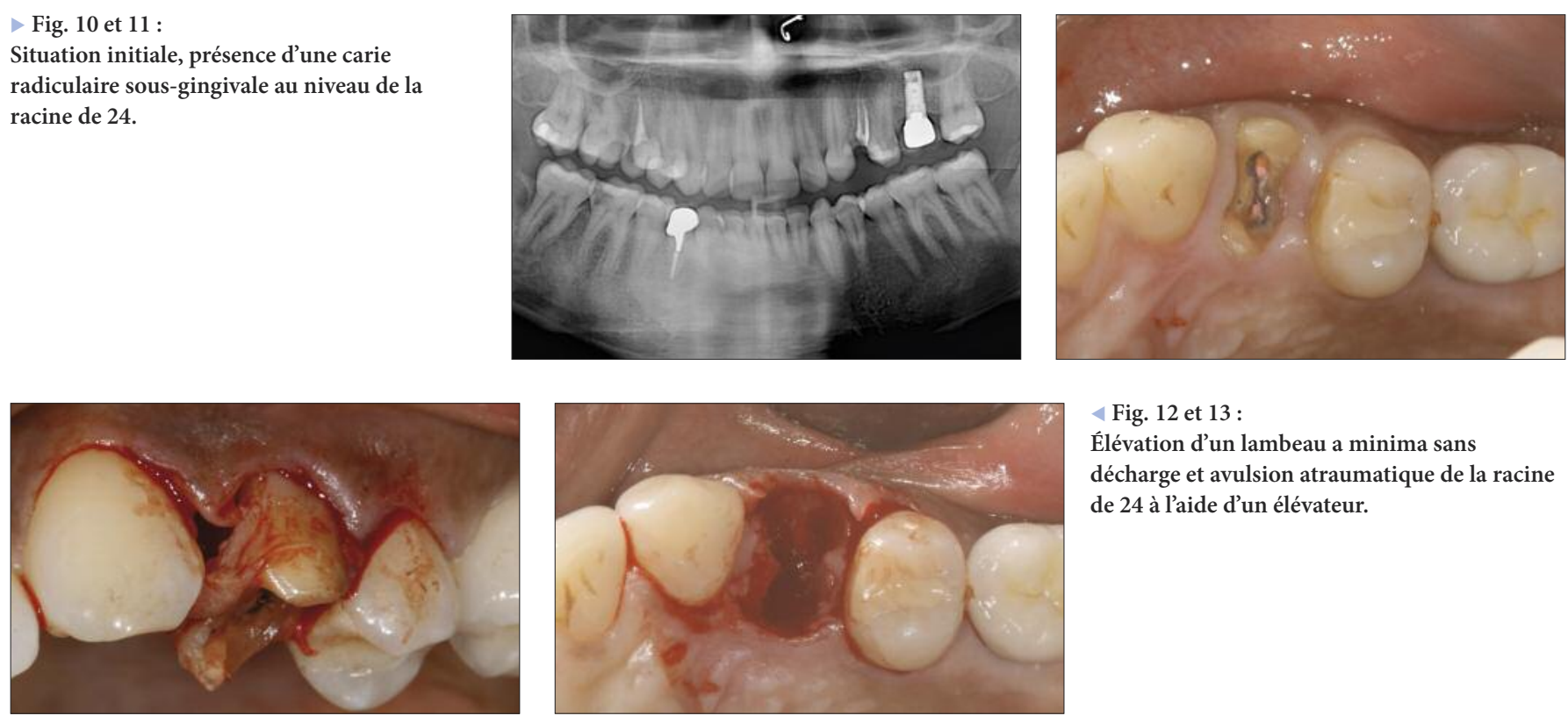

4 Fig. 12 et 13 :

Élévation d'un lambeau a minima sans décharge et avulsion atraumatique de la racine de 24 à l'aide d'un élévateur.
$>$ Fig. 14:

Forage selon le guidage apicopalatin et mise en place d'un implant Serf EVL 4/10 à distance de la paroi vestibulaire.
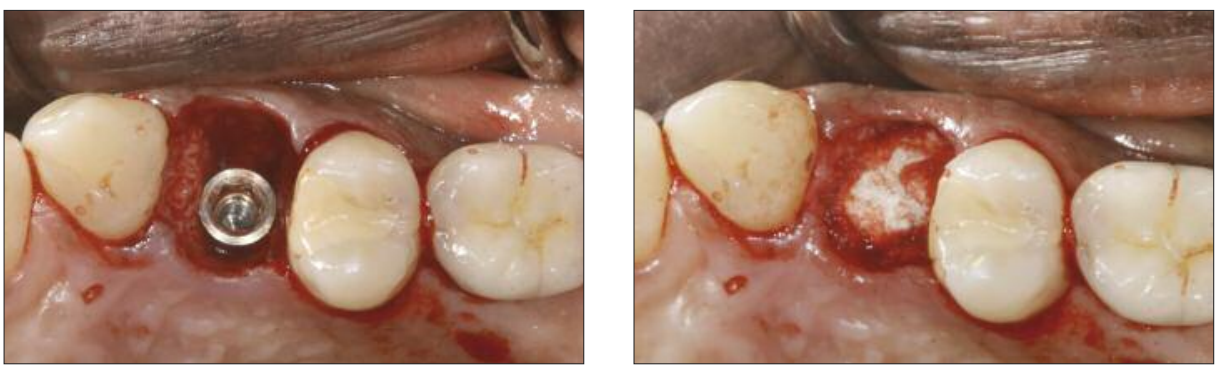

4 Fig. 15 :

Le hiatus entre l'implant et la paroi vestibulaire n'est pas comblé. Une membrane de collagène recouvre l'implant avec sa vis de couverture, elle a pour objectif de stabiliser le caillot sanguin. 
Implantologie Leclercq P., Granjon O., Phillips D.

\section{Cas clinique $n^{\circ} 1$ (suite)}

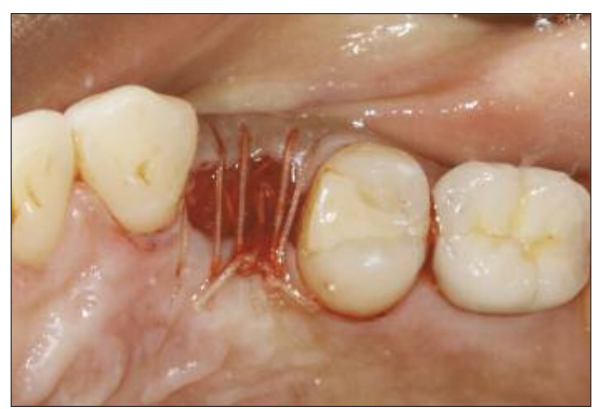

$\triangleleft$ Fig. 16 :

Le lambeau est

suturé sans chercher

à obtenir une

fermeture

hermétique du site.

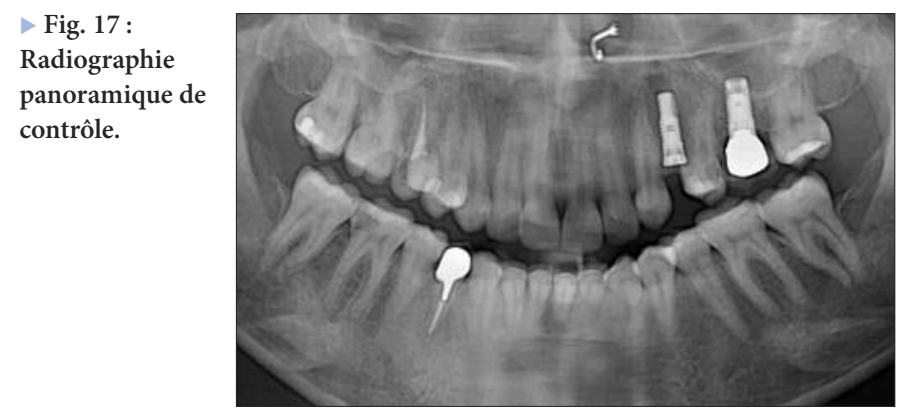

Fig. 18 :

Cicatrisation à trois

mois, le site s'est

complètement

refermé.

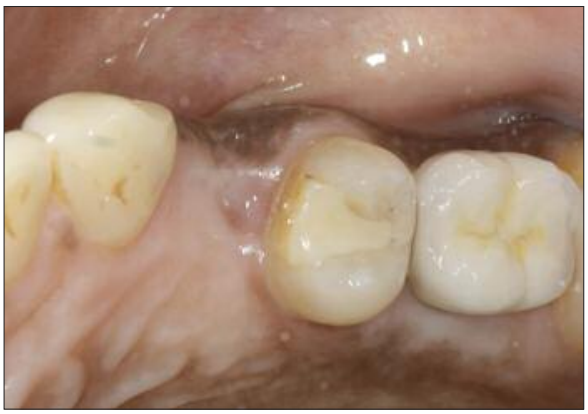

$\triangleleft$ Fig. 20 :

Vue occlusale, le profil d'émergence a été mis en forme grâce à la prothèse transitoire.

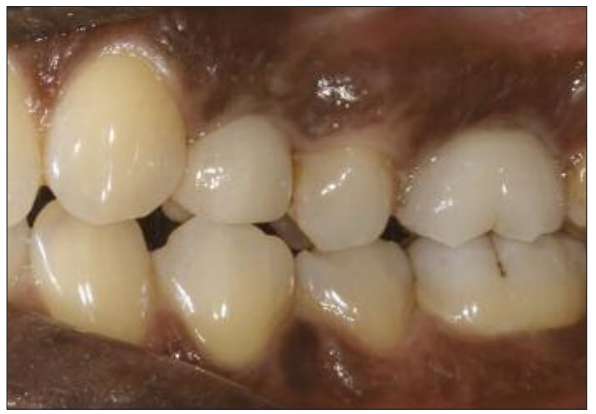

$\triangle$ Fig. 19:

Mise en charge de

l'implant grâce à une prothèse transitoire transvissée.
> Fig. 21 :

Trois ans après la pose de la couronne

céramo-métallique, les papilles ont comblé les espaces interdentaires.
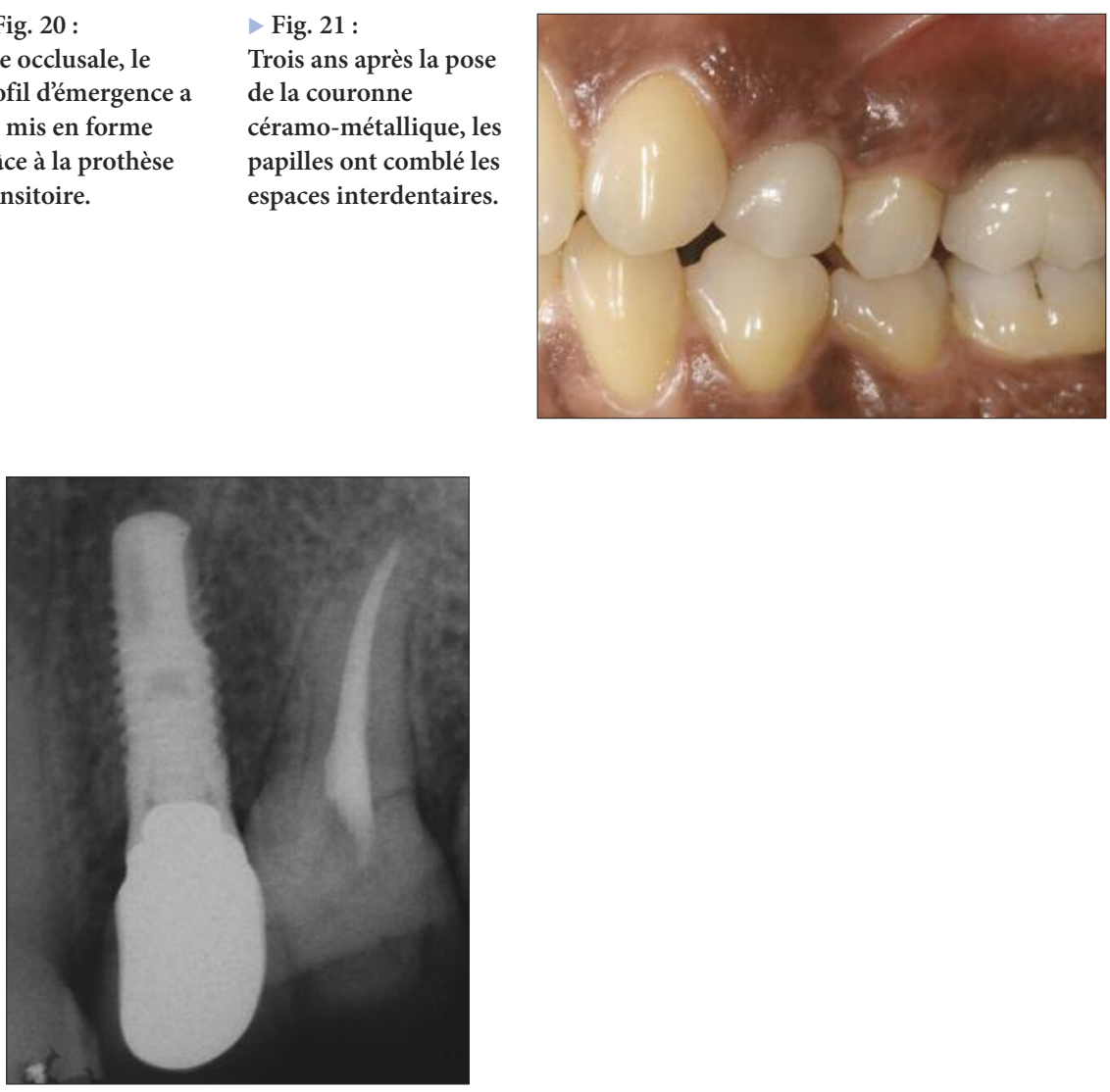

$\triangle$ Fig. 22 :

Radiogaphie rétro-alvéolaire de contrôle à 3 ans. 


\section{Cas clinique $\mathrm{n}^{\circ} 2$}

> Fig. 23 :

Chez ce patient, la

22 est fortement égressée et présente une mobilité 3. La dent doit être avulsée.
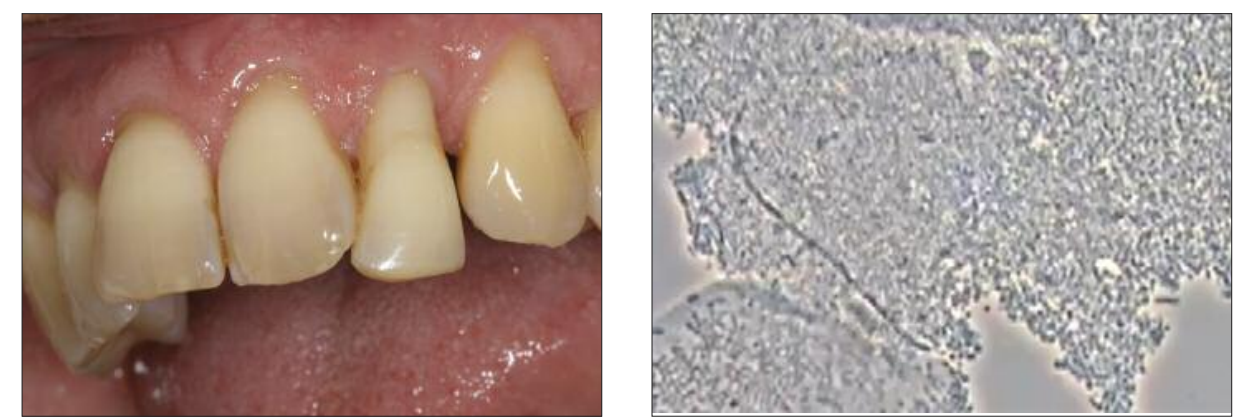

$\triangleleft$ Fig. 24 :

Une analyse au

microscope à contraste de phase de la plaque dentaire prélevée au niveau de cette dent met en évidence la présence d'une flore microbienne saine.

\footnotetext{
> Fig. 25 et 26 : Avulsion de 22 et réalisation d'un lambeau a minima.
}
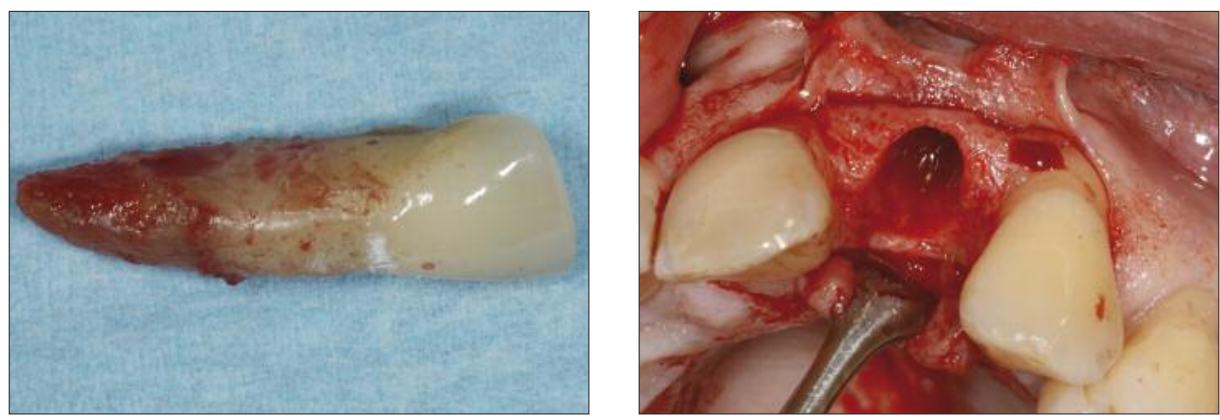

Fig. 27 :

Mise en place d'un implant Serf EVL de diamètre 4 et longueur 10 au contact de la paroi palatine. Le lambeau sera suturé sans traction et sans combler le hiatus entre l'implant et la paroi vestibulaire de l'alvéole.
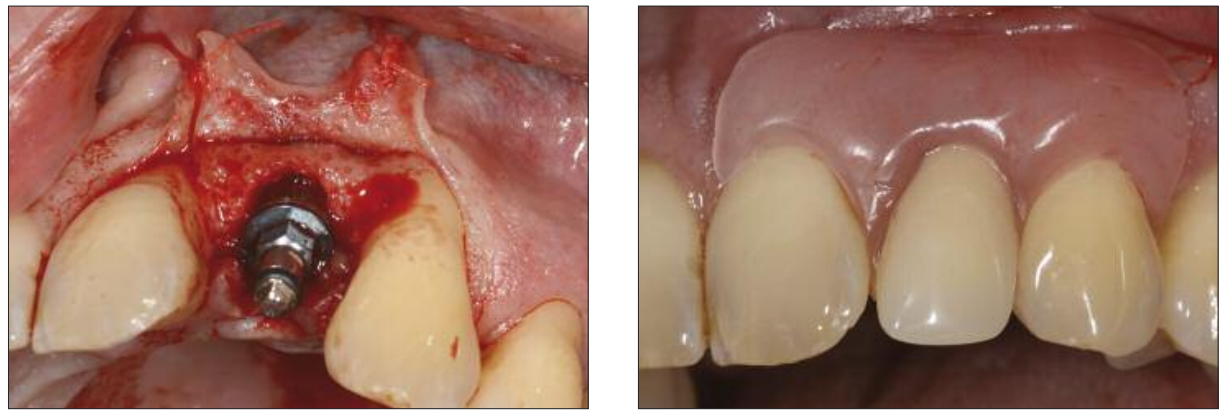

4 Fig. 28 : Mise en place d'une prothèse amovible de temporisation en nylon.

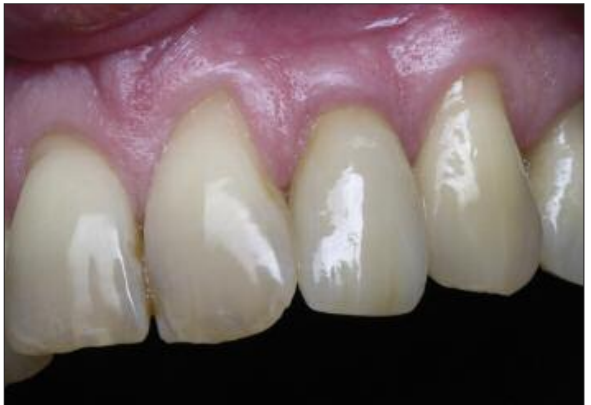

$\triangle$ Fig. 29 :

Contrôle à 4 ans, la prothèse d'usage a été réalisée par le docteur Christine MASSINON en collaboration avec Monsieur Régis LEPAITTRE.

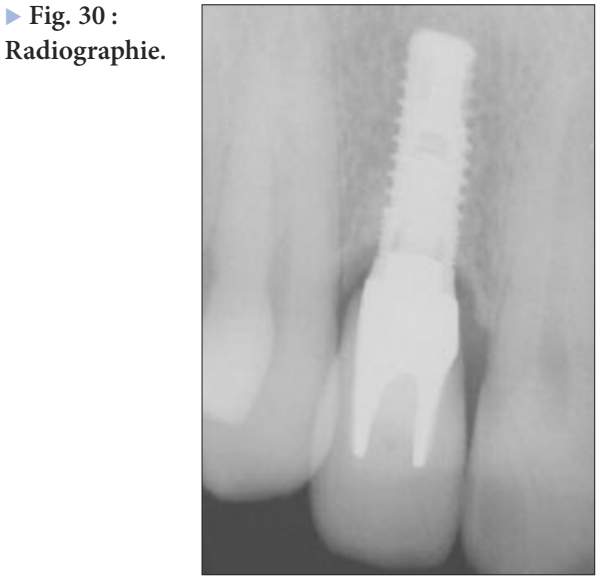

Fig. 30 : Radiographie.

\section{CONCLUSION}

L'AII répond à la demande de plus en plus fréquente de nos patients qui souhaitent minimiser le nombre d'interventions et réduire le temps de traitement. Toutefois, il n'est pas question de généraliser cette technique car la gestion des situations d'échec est souvent beaucoup plus complexe qu'avec une technique classique. De ce fait, l'AII ne s'adresse pas à des praticiens débutants mais doit être mise en œuvre par des praticiens expérimentés qui en connaissent les indications et les contre-indications. 


\section{Bibliographie}

[1] Esposito M, Grusovin MG, Polyzos IP, Felice P, Worthington HV. Timing of implant placement after tooth extraction immediate, immediate-delayed or delayed implants? A Cochrane systemic review. Eur J Oral Implantol 2010;3(3):189-205.

[2] Siebers D, Gehrke P, Schliephake H. Delayed function of dental implants: a 1- to 7-year follow-up study of 222 implants. Inter J Oral Maxillofac Implants 2010;25(6):1195-202.

[3] Polizzi G, Grunder U, Goené R, Hatano N, Henry P, et al. Immediate and delayed implant placement into extraction sockets: a 5-year report. Clinical Implant Dentistry and Related Research 2000;2(2)93-9.

[4] Botticelli D, Berglundh T, Lindhe J. Hard-tissue alterations following immediate implant placement in extraction sites. J Clin Periodontol 2004;31(10) 820-8.

[5] Araújo MG, Wennström JL, Lindhe J. Modeling of the buccal and lingual bone walls of fresh extraction sites following Implant installation. Clin Oral Impl Res 2006;17(6):606-14.
[6] Novaes AB Jr, Papalexiou V, Grisi MF, Souza SS, Taba M Jr, Kajiwara JK. Influence of implant microstructure on the osseointegration of immediate implants placed in periodontally infected sites. A histomorphometric study in dogs. Clin Oral Implants Res 2004;15(1):34-43.

[7] Novaes AB Jr, Marcaccini AM, Souza SL, Taba M Jr, Grisi MF. Immediate placement of implants into periodontally infected sites in dogs: a histomorphometric study of bone-implant contact. Int J Oral Maxillofac Implants 2003;18(3):391-8.

[8] Waasdorp JA, Evian CI, Mandracchia M. Immediate placement of implants into infected sites: a systematic review of the literature. J Periodontol 2010;81(6):801-8. Review.

[9] Bonner M. Entamoeba gingivalis dans les parodontites. Inf Dent 2003;85(24):1659-66.

[10] Tarnow DP, Cho SC, Wallace SS. The effect of inter-implant distance on the height of inter-implant bone crest. J Periodontol 2000;71(4):546-9.

[11] Hwang KG, Park CJ. Ideal implant positioning in an anterior maxillary extraction socket by creating an apicopalatal guiding slot: a technical note. Int $J$ Oral Maxillofac Implants 2008;23(1):121-2.

[12] Chen ST, Darby IB, Reynolds EC, Clement JG. Immediate implant placement postextraction without flap elevation. J Periodontol 2009;80(1):163-72.

[13] Tarnow DP, Chu SJ. Human histologic verification of osseointegration of an immediate implant placed into a fresh extraction socket with excessive gap distance without primary flap closure, graft, or membrane: a case report. Int J Periodontics Restorative Dent 2011;31(5):515-21.

[14] Smith RB, Tarnow DP, Brown M, Chu S, Zamzok J. Placement of immediate implant and a fixed provisional restoration to replace the four mandibular incisors. Compend Contin Educ Dent 2009;30(7):408-16.

[15] Missika P, Bert M, Tavernier B, Fromentin O. 30 questions en implantologie. Paris : Espace ID Presse, Édition Multimédia, 2011.

\section{$\operatorname{aOs}=$

\section{Vous souhaitez publier un article dans les Actualités Odonto-Stomatologiques}

nouvelle formule?

Pour publier votre article original et être lu par l'ensemble des praticiens français, soumettez-le au comité de rédaction des AOS.

Téléchargez les instructions aux auteurs sur www.aos-journal.org Envoyez votre article à secretariat.aos@edpsciences.org 